\title{
Bases for maternal perceptions of infant crying and colic behaviour
}

\author{
Ian St James-Roberts, Susan Conroy, Katie Wilsher
}

\begin{abstract}
According to the commonest definition, infant colic is distinguished by crying which is 'paroxysmal'-that is, intense and different in type from normal fussing and crying. To test this, maternal reports of the distress type of 67 infants whose fuss/crying usually exceeded three hours a day ('persistent criers') were scrutinised using 24 hour audiorecordings of the infants' distressed vocalisation. 'Moderate criers'( $n=55)$ and 'evening criers' $(n=38)$ were also assessed.
\end{abstract}

Most of the distress in all three groups was fussing. In the audiorecordings the persistent criers showed a higher crying: fussing ratio than the moderate criers, but intense crying was rare. A third of the persistent criers were reported by their mothers to have occasional, distinct colic bouts of 'intense, unsoothable crying and other behaviour, perhaps due to stomach or bowel pain.' In the audiorecordings these periods were longer, but not paroxysmal in onset or more intense than the crying of persistent criers not judged to have colic.

The audible features of the crying may be less important than its unpredictable, prolonged, hard to soothe, and unexplained nature.

(Arch Dis Child 1996;75:375-384)

Keywords: infant crying, colic, maternal perceptions.

In Western societies bouts of prolonged, unexplained infant crying in the first few months are a common source of concern for parents. ${ }^{12}$ The crying has been linked to parental distress and, in rare instances, to infant abuse. ${ }^{34}$ Two United Kingdom studies have found that about one in six families with infants of this age approach health visitors or general practitioners because of their baby's excessive crying, ${ }^{25}$ making this a costly complaint for the health services. There is objective evidence that such parents are correct in believing that their babies cry substantially more than average. ${ }^{5} \mathrm{At}$ present, there are no agreed primary health care policies or methods for managing the crying.

For many years this phenomenon has been widely known as 'colic.' This term is often used descriptively, to identify infants who cry a lot or whose parents consult health professionals as a result. ${ }^{6}{ }^{7}$ However, the word 'colic' also implies an explanation for the crying. As Carey $^{8}$ has pointed out, it derives from the
Greek word for the intestine. For Illingworth ${ }^{9}$ and many others, the term denotes a digestive disorder which gives rise to pain, causing the infant to cry. In this sense the word colic signifies a discrete clinical syndrome, distinct from normal infant fussing and crying. Consistent with this, Wessel and colleagues' ${ }^{10}$ widely used definition of colic refers to 'paroxysms of irritability, fussing or crying lasting for a total of more than three hours a day and occurring on more than three days in any one week.' The word 'paroxysms' seems to imply that colic behaviour is different from normal fussing and crying: it is more intense, with a sudden onset, and harder to placate, presumably because of the underlying discomfort or pain. Similarly, in a review of 50 articles on colic, Barr ${ }^{11}$ found that $42 \%$ of them included a paroxysmal nature as a feature of colic bouts.

As well as this clinical approach, a nonclinical explanation of infant crying in the early months has been suggested by studies of infant crying in the general community. ${ }^{212}{ }^{13}$ These have found that fussing and crying in Western infants increase from birth to reach an average of about two hours a day at around 6 weeks of age, the peak age for crying. Around a fifth to a quarter of infants fuss and cry for three or more hours a day at this age. The crying contains a striking evening clustering, so that crying between 1800 hours and midnight makes up about $40 \%$ of the daily total. Both the evening clustering and crying generally decline after 3 months of age, so that infants cry around half as much in months 4 to $12 .^{2}$

These findings suggest that the early crying peak is a normal feature of infant development, perhaps due to maturational changes which occur at this age,$^{14}$ rather than to a clinical disorder. Some support for this developmental approach comes from studies, including two in non-Western societies, which have found evidence of the crying peaks in spite of substantial variations in parental care. ${ }^{15-17}$ According to this viewpoint, infants labelled as colic cases are simply at the extreme tip of the normal distribution of crying. In support of this, there is evidence that parents most often seek professional help for 'excessive' crying at around the peak age for crying in infants generally. ${ }^{2}$

Which of these explanations is correct, or whether two phenomena and groups of infants exist, are questions of practical, as well as theoretical, importance. Whereas the colic approach implies the need to identify and treat a gastrointestinal disturbance in a small number of infants, the developmental viewpoint implies 
that interventions should be aimed at providing parents with information and support, so as to help them to contain and cope with the crying. ${ }^{18}$

One way to differentiate the colic and developmental explanations of early infant crying is to determine whether the crying of infants who cry a lot at this age is paroxysmal, particularly intense, or otherwise distinct from normal infant crying, as the colic explanation supposes. To address this issue, a recent maternal diary study explored whether infants, selected using the Wessel et al criteria for crying amount, differed from other infants in their type of crying. ${ }^{19}$ This study found that colic behaviour, defined as 'bouts of intense, unsoothable crying and other behaviour, perhaps due to stomach or bowel pain,' was reported more often in infants selected using the Wessel criteria than in other infants. However, two thirds of the distress reported in the selected infants was fussy, fretful behaviour, together with a small amount of crying, and most of these infants did not show any colic behaviour at all. About one third of the mothers of the Wessel infants did identify distinct periods of colic behaviour. On average, one in six of these infants' distress periods was considered to be a colic bout.

These findings indicate that most infants selected using the Wessel criteria for overall fussing and crying amount, do not exhibit colic behaviour as defined. They also suggest that some of the mothers of these infants perceive colic behaviour as a distinct and rare type of distress. A question raised is how far these mothers' perceptions reflect objectively measurable features of infant behaviour, and what these are. Deciding that a particular bout of distress is distinct involves complex subjective judgments, so that the bases for, and reliability of, the judgments need to be examined.

Using audiorecordings of infant vocalisation this study aimed to provide a detailed analysis of the characteristics which differentiated the crying and colic bouts these mothers identified.

\section{Methods}

SAMPLE RECRUITMENT AND SCREENING

Over 11 successive months, about 50 new mothers were approached each month in a large maternity hospital in London. Twins and infants admitted to intensive care were excluded, but, otherwise, the mothers were approached unselectively. Of 597 mothers contacted, 558 (93\%) agreed to participate in the study. When each infant reached 4 to 5 weeks of age, a structured maternal telephone interview, based on the Crying Patterns Questionnaire, $(\mathrm{CPQ})^{2}$ was used to screen the infants for the number of minutes they fussed and cried in each part of the day: morning $(0600$ hours to noon); afternoon (noon to 1800 hours); evening (1800 hours to midnight); night (midnight to 0600 hours). The mothers were asked to identify the amounts of fussing and crying per time of day that had occurred on most days in the last week. Interview data were successfully obtained for 530 infants ( $89 \%$ of those approached in hospital).

\section{SELECTION AND ASSESSMENT OF SUBGROUPS}

Using the CPQ screening measures, three groups of infants were chosen for follow up assessments: Persistent criers $(\mathrm{n}=67)$, who met slightly modified Wessel criteria by fuss/crying for three or more hours per day most days in the last week. Evening criers $(\mathrm{n}=38)$, who fuss/cried for an hour or more in the evenings, 30 minutes or less in every other period of the day, and less than three hours in total. Moderate criers $(n=55)$, who fuss/cried for 30 minutes or less in all four periods of the day.

The demographic particulars of the 160 successfully selected cases were compared with those of the screening sample and with United Kingdom norms. ${ }^{19}$ There were no demographic differences among the crying groups. However, compared with United Kingdom mothers in general, those who participated in the home assessments were slightly older, of a higher social class, and less likely to be of Asian or Afro-Caribbean origins, while the assessed infants were more likely to be firstborns.

At 5 to 6 weeks of age, the 160 selected infants were followed up at home, where 24 hour diaries were used to confirm the CPQ figures. According to the method of Hunziker and Barr, ${ }^{13}$ the diaries were completed prospectively by the mothers, such that infant behaviours (feeding, sleeping, awake content, distress) were shaded in, more or less as they occurred, on charts, with a resolution of 5 minutes of time. For this study, the mothers were asked to shade in three types of distress: fussing; crying; and colic separately, using the following definitions: Fussing: 'baby is unsettled and irritable and may be vocalising but not continuously crying.' Crying: 'periods of prolonged distressed vocalisation.' Colic: 'bouts of intense, unsoothable crying and other behaviour, perhaps due to stomach or bowel pain.'

The diaries were kept for three successive days wherever possible ( $82 \%$ of the 160 mothers kept them for at least one day, $72 \%$ for two days, and $56 \%$ for three days. Eighteen per cent of the mothers, evenly divided between the groups, failed to provide diary data). When a period of colic was entered on the diary, the mother also completed a behaviour checklist, modelled on Lester et al's ${ }^{20}$ Colic Symptom Checklist, where she checked the particular behaviours she had observed to be evidence of colic. The checklist included crying features, such as sudden onset or sounding intense or painful, as well as visible behaviours, such as bringing up the knees. In all, 23 possible behaviours, drawn from colic studies, could be checked, and mothers could add others.

As well as these maternal measures, a 24 hour audiorecording of each infant's vocalisations was made during one of the diary days, using calibrated radio microphones and voiceactivated tape recorders, as in previous research. ${ }^{5}$ The circuitry included an electronic speaking clock, which recorded the onset time of each vocalisation, and audible clicks at one second intervals throughout the vocalisation 
on the second audiotape channel, allowing precise measures of the time of day and duration of each period of vocalisation. All the parents were also offered an appointment with a consultant paediatrician, who gave the infants a health, growth, and development check. The study was approved by the hospital ethics committee.

DATA TRANSCRIPTION AND PROCESSING

The diaries were entered into the computer by researchers unaware of the infant's group, to provide figures for the length and number of behaviour periods. Summary measures of the total number of minutes of fuss, crying, and colic which occurred over 24 hours were also derived. Lastly, composite distress figures were obtained by totalling the number of minutes of fuss + crying + colic over 24 hours.

The audiorecordings were listened to and transcribed on to printed record sheets by blinded, trained researchers. Only distressed vocalisation was transcribed, such that each successive five seconds of distressed vocalisation were coded separately as one of four types of distress, using the following definitions: Fussing: distressed vocalisation which is discontinuous, quieter than crying within an individual baby, and a shallow sound produced from the throat. Borderline: distressed vocalisation which is inbetween fussing and crying; either (a) quiet in intensity, but more continuous than fussing, or (b) loud in intensity, but not continuous. Crying: distressed vocalisation which continues throughout the $\mathbf{5}$ seconds, is louder than fussing within each baby, and a sound produced using a fuller expansion of the lungs than fussing. Intense crying: distressed vocalisation that is both loud and frenzied sounding, conveying an urgent or imperative signal.

Both the written definitions and standard 'template' audiorecordings of each type of distress vocalisation were used in training and during the transcriptions to enhance reliability. The 'borderline' category was also included to increase reliability, allowing distressed vocalisation of this type to be combined either with fussing or crying during data analysis.

Measures of the number of seconds of fussing, borderline, crying, intense crying and overall distress 'were obtained from the transcription sheets. Following the format of previous studies, ${ }^{5} 19$ two summary measures of distressed vocalisation were derived. The first, vocalisation duration measure, was a simple total of the number of seconds of distressed vocalisation, and of each vocalisation type, included in a recording. Although this measure is objective, it has the disadvantage that it underrepresents the amounts of distress parents record on diaries. Distressed infant vocalisation typically occurs in sequences, usually called episodes, bouts, or periods. ${ }^{21}$ The periods have an inherent burst and pause structure, such that bursts of vocalisation are punctuated by frequent short pauses, presumably to allow respiration and to maintain metabolism within bounds. To accommodate this, a second objective measure, of distress period duration, can be derived. This measure reflects the lengths of distress periods, including both the distressed vocalisations and the intervening pauses. As parents in everyday settings are unlikely to identify the brief pauses, this measure probably comes closer to what parents perceive. Such distress periods, together with their start and stop times, are readily discernible on the audiorecording transcription sheets. However, to ensure uniformity and to obtain measures which corresponded as closely as possible to the diary measures, a five minute rule was used to define the lengths of distress periods, as in previous research. ${ }^{519}$ Following this, a 5 minute 'moving window' was passed across each audiotape transcription sheet. Where 10 seconds or less of distressed vocalisation occurred within a 5 minute segment, this was considered an isolated instance and was disregarded. Where 15 seconds or more occurred, the window was moved until a continuous 5 minute segment without distress was found, signifying the end of the distress period. The lengths of such distress periods and minutes of fussing, borderline, crying, intense crying and intervening pauses were then computed and totalled over 24 hours.

To confirm the reliability of the resulting measures, 16 (10\%) of the 157 recordings were transcribed independently by two researchers and their measures of vocalisation duration, distress period duration, and minutes of time spent in each type of distress, were compared. The discrepancy, which can be interpreted as a measure of measurement error, was less than $2 \%$ for each of the measures reported.

\section{Results}

Unless otherwise indicated, the diaries analysed in the present study are for the 24 hour period which corresponded to the audiorecording. The analyses are presented in four stages. First, the three main groups were compared on the audiorecording and diary measures of amounts and types of distress. Next, the infants were subdivided to compare infants reported to show colic behaviour with other infants. Third, whether periods identified as 'colic' have a paroxysmal onset was explored. Lastly, the mothers' colic checklist findings were examined to clarify the bases for their reports.

COMPARISON OF DIARY WITH AUDIORECORDING MEASURES OF AMOUNTS AND TYPES OF DISTRESS Table 1 summarises the audiorecording vocalisation, audiorecording period, and diary measures of the 24 hour total minutes of distress for the three groups of infants. As expected, the audiorecording measures of audible distress vocalisation showed much less distress than the audiorecording period measures. Periods of infant distress were made up chiefly of the non-vocal pauses which occur between vocalisations, rather than actual distress vocalisation. Presumably, these pauses included non-vocal distressed behaviour. The audiorecording period measures were close to the amounts of 
Table 1 Comparison of audio recording with diary measures of amounts of distress in three groups of infants studied

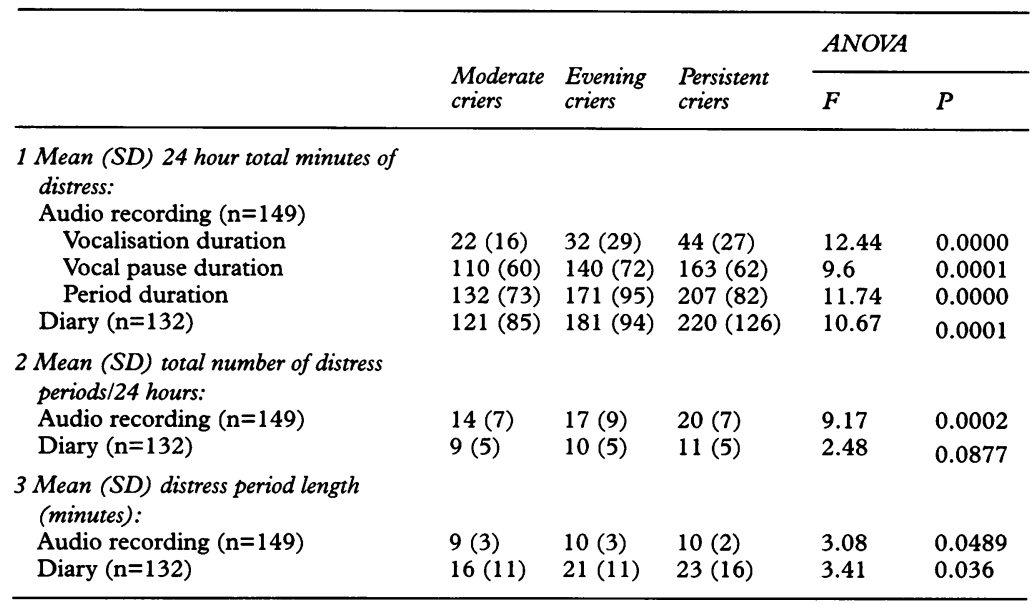

Audio: moderate criers: $n=53$; evening criers: $n=35$; persistent criers: $n=61$.

Diary: moderate criers: $n=43$; evening criers: $n=31$; persistent criers: $n=50$.

distress the parents reported in their diaries. The mean 24 hour audiorecording period and diary totals were within 15 minutes of each other for each of the groups (table 1), indicating that parental reports are based on distress periods rather than minutes of vocal distress. However, the group differences were essentially the same, irrespective of the measurement method. As table 1 shows, each of the measures showed highly significant group differences, such that the evening criers' distress totals fell inbetween those of the persistent and moderate criers. As intended, the mean 24 hour distress totals for the persistent criers substantially exceeded three hours a day on both the diary and audiorecording period measures. Correlational analyses showed significant correlations of 0.6 to 0.7 between the diary and both audiorecording vocalisation and audiorecording period measures. Others ${ }^{22}$ have reported similar correlations between diary and audiorecording vocalisation measures of 10 infants.

Table 1 also summarises the measures of distress period length and frequency derived from the diary and audiorecording methods. Consistent with the findings of Barr et $a l,{ }^{7}$ the main group difference in the diaries was in the length of distress periods, rather than the number. Persistent criers had longer, rather than more frequent, distress periods according to parental diaries. However, the audiorecording period measures identified significant group differences both in the length and frequency of distress periods. This is probably because the audiorecording period measures identify more frequent, shorter distress periods than the diaries. Presumably, mothers sometimes overlook silences of 5 minutes or more and combine two periods of distress, either because they perceive non-vocal distress during the interval, or because they ignore some intervals without distress. It may be possible to combine the audiorecording period measures in a similar way, but, in the absence of information about the basis for maternal decisions, no attempt to combine the periods was made here.
The first variable in table 2 represents the proportion of the total audiorecorded distress period time which was filled with audible distress vocalisation. Although this ratio does not distinguish distress type, it provides a simple and objective index of the density or intensity of the infants' distressed vocalisation. The persistent criers' distress was characterised by a higher percentage of vocalisation within distress periods on the audiorecording measures.

Table 2 also summarises the findings for each type of distress found in each group by each method. To simplify the audiorecording measures, borderline vocalisation in any distress period was recoded as fussing or crying, according to which of these occurred most in that period. The audiorecording vocalisation figures in table 2 are, then, the proportion of the 24 hour total minutes of distressed vocalisation that consisted of fussing, crying, or intense crying. Comparison of the audiorecording period with diary measures has to take into account that mothers usually categorised each distress period as a single type (fussing, crying, or colic), whereas many periods transcribed from the audiorecordings consisted of a mixture of fussing and crying. There were no audiorecorded periods where intense crying was the only type of distress. To allow the findings to be compared, a 'majority rule' was applied to the audiorecording period data. Each period was examined for the predominant type of distress and the period was categorised accordingly as fussing, crying, or intense crying.

Although it is not clear whether mothers use similar decision rules in typing distress periods, table 2 shows that these algorithms produce a substantial correspondence between the diary and audiorecording methods. According to the maternal diaries, fussing was the principal type of distress in each group of infants, with crying making up a quarter to a third of distressed vocalisation, overall. Colic bouts were rare, comprising $0.6 \%, 6 \%$, and $8 \%$, respectively of the overall distress of moderate, evening, and persistent criers. With some variation due to the methods of calculation involved, the audiorecording vocalisation and period measures both confirm that fussing was the majority type of distress for all three groups of infants, while crying made up $24-45 \%$ of each group's distress total. The audiorecordings did not measure colic behaviour. However, intense crying was rare, comprising $3 \%, 3 \%$, and $4 \%$ of the distress vocalisation and $3 \%, 1 \%$, and $2 \%$ of the distress periods of moderate, evening, and persistent criers, respectively. These figures also show that the method used to categorise the audiorecorded distress periods did not substantially underestimate the amounts of intense crying.

The analysis of variance (ANOVA) and Duncan multiple range significance tests for each group pair in table 2 confirm that, on both the diary and audiorecording measures, the persistent criers exhibited proportionately less fussing than the moderate criers. The diary measures showed no group differences in the 
Table 2 Comparison of audio recording with diary measures of proportions of each type of distress per 24 hours

\begin{tabular}{|c|c|c|c|c|c|c|c|c|c|}
\hline & \multicolumn{2}{|c|}{ Moderate criers } & \multicolumn{2}{|c|}{ Evening criers } & \multicolumn{2}{|c|}{ Persistent criers } & \multicolumn{2}{|c|}{ ANOVA } & \multirow{2}{*}{$\begin{array}{l}\text { Duncan } \\
\text { MR test }\end{array}$} \\
\hline & $\%$ & $(S D)$ & $\%$ & $(S D)$ & $\%$ & $(S D)$ & $F$ & P test & \\
\hline \multicolumn{10}{|l|}{ Audio recording distress vocalisation $(n=149)$ : } \\
\hline Vocalisation minutes as \% period duration & 15 & (7) & 17 & (7) & 20 & (7) & 8.3 & 0.0004 & $\mathrm{P}>\mathrm{EM}$ \\
\hline Fussing minutes as $\%$ vocalisation & 67 & (27) & 67 & (21) & 52 & (21) & 7.88 & 0.0006 & $\mathbf{P}<\mathrm{EM}$ \\
\hline Crying minutes as \% vocalisation & 30 & (23) & 30 & (19) & 45 & (18) & 9.6 & 0.0001 & P $>$ EM \\
\hline Intense crying minutes as $\%$ vocalisation & 3 & (7) & 3 & (6) & 4 & (6) & 0.1 & 0.9 & NS \\
\hline \multicolumn{10}{|l|}{ Audio recording distress period $(n=149)$ : } \\
\hline Fussing bouts as $\%$ period duration & 73 & (28) & 74 & (24) & 60 & (24) & 5.12 & 0.007 & $\mathrm{P}<\mathrm{EM}$ \\
\hline Crying bouts as \% period duration & 24 & (24) & 24 & (24) & 38 & (22) & 6.14 & 0.003 & $\mathrm{P}>\mathrm{EM}$ \\
\hline Intense crying bouts as \% period duration & 3 & (10) & 1 & (4) & 2 & (6) & 0.5 & 0.61 & NS \\
\hline \multicolumn{10}{|l|}{ Diary measures $(n=132)$ : } \\
\hline Fussing minutes as $\%$ distress & 74 & (26) & 64 & (29) & 59 & $(22)$ & 4.34 & 0.015 & $\mathbf{P}<\mathbf{M}$ \\
\hline Crying minutes as \% distress & 26 & (26) & 30 & (25) & 33 & $(21)$ & 1.2 & 0.31 & NS \\
\hline Colic minutes as $\%$ distress & 0.5 & (4) & 6 & (16) & 8 & (16) & 4.1 & 0.018 & $\mathbf{P}>\mathbf{M}$ \\
\hline
\end{tabular}

Sample sizes as in table 1.

proportion of crying, so that according to this method, the persistent criers' reduced fussing was matched by an increase in the proportion of colic behaviour. In contrast, on both audiorecording measures, the reduction in fussing was matched by a significant increase in the proportion of crying. There were no group differences in the proportions of audiorecorded intense crying. Broadly speaking, the evening criers resembled the moderate criers, or fell between these and the persistent criers, on these measures of their distress type.

In summary, allowing for variations of definition and method, these findings support the validity of maternal reports that infants who cry persistently differ from other infants in their distress type, as well as amount. Compared with the moderate criers, in particular, proportionately less of the persistent criers' distress was fussing and proportionately more was crying (audiorecording measures) or colic behaviour (maternal diaries).

\section{DISTINGUISHING COLIC FROM OTHER DISTRESS} PERIODS

Based on the 'rule of threes' definition of amount of fussing and crying, all the persistent criers selected for this study could be considered colic cases. However, using the explicit definition of colic behaviour defined here, only 20 of $54(35 \%)$ persistent criers, eight of 33 (24\%) evening criers, and three of $45(7 \%)$

Table 3 Distribution of colic $v$ non-colic distress periods recorded in diaries

\begin{tabular}{|c|c|c|c|c|}
\hline & \multicolumn{2}{|l|}{ Colic infants } & \multirow{2}{*}{$\begin{array}{l}\text { Other persistent } \\
\text { criers' fuss/cry } \\
\text { periods }\end{array}$} & \multirow{2}{*}{$\begin{array}{l}\text { Evening/ } \\
\text { moderate criers' } \\
\text { fuss/cry periods }\end{array}$} \\
\hline & Colic periods & Fuss/cry periods & & \\
\hline \multicolumn{5}{|l|}{ Start time: } \\
\hline Midnight -0800 & $24 \%$ & $20 \%$ & $16 \%$ & $19 \%$ \\
\hline $0800-1600$ & $27 \%$ & $39 \%$ & $38 \%$ & $37 \%$ \\
\hline 1600-midnight & $49 \%$ & $41 \%$ & $46 \%$ & $44 \%$ \\
\hline \multicolumn{5}{|l|}{$\begin{array}{l}\text { Immediately previous period } \\
\text { type: }\end{array}$} \\
\hline Sleep & $12 \%$ & $42 \%$ & $39 \%$ & $39 \%$ \\
\hline Feed & $41 \%$ & $25 \%$ & $20 \%$ & $20 \%$ \\
\hline Awake & $13 \%$ & $34 \%$ & $41 \%$ & $41 \%$ \\
\hline Fuss + /or cry & $34 \%$ & - & - & - \\
\hline $\begin{array}{l}\text { Mean (SD) minutes since } \\
\text { previous sleep period }\end{array}$ & $66(70)$ & $44(58)$ & $52(75)$ & $45(79)$ \\
\hline $\begin{array}{l}\text { Mean (SD) minutes since } \\
\text { previous feed period }\end{array}$ & $45(67)$ & $80(87)$ & $75(84)$ & $78(82)$ \\
\hline
\end{tabular}

Colic infants: $n=31$; other persistent criers: $n=33$; other/evening/moderate criers: $n=63$; colic infants' colic periods: $n=140$; colic infants' non-colic fuss + /or cry periods: $n=587$; other persistent criers' fuss + /or cry periods: $n=655$; other evening/moderate criers' fuss $+/$ or cry periods: $\mathrm{n}=1075$. moderate criers had any colic behaviour in their diaries. For these infants, one in six of their distress periods, on average, was identified as a colic bout.

Of all the diaries available, 140 colic periods were reported for these 31 colicky infants. In just over half $(51 \%)$ of cases, these colic periods were not directly preceded or followed by other types of distress: the whole distress period was designated as colic behaviour. However, $49 \%$ of the colic periods were directly preceded or followed by periods containing fussing and/or crying. In almost all such cases, the distress began with fussing and/or crying, which then led on directly to colic behaviour. To assess the characteristic features of the colic periods, their distribution over the day and in relation to feeding and sleeping periods was mapped, compared with the non-colic periods of distress of the same infants. Table 3 summarises the results. To aid the comparison, the equivalent figures for persistent criers, and moderate/evening criers, not reported to have colic, are also shown. Colic periods resembled distress periods more generally in having their peak onset between 1600 hours and midnight. Colic periods were more likely to follow feeds and less likely to follow sleep than non-colic distress periods. In $41 \%$ of cases, colic periods followed directly after feeds while, if the analysis is based on periods of distress containing colic, $51 \%$ of such periods followed feeds directly. In comparison, only $20-25 \%$ of distress periods without colic did so. The mean latency from feeds to colic periods, 45 minutes, was also substantially less than the latency of 80 minutes for distress periods without colic. This remained true even when the comparison was based solely on distress periods which did not follow feeds directly. These findings suggest some contingency between feeding and colic periods. However, they also exhibit considerable variability, so that, for example, $27 \%$ of colic periods began more than an hour after the previous feed.

Of the 31 infants reported to show colic behaviour, corresponding diaries and audiorecordings were available for 14 infants. In the remainder, the colic periods identified on the diaries occurred on days which were not audiorecorded. Between them, the 14 infants had 26 colic periods recorded on their diaries (table 
Table 4 Length of colic periods compared with non-colic periods

\begin{tabular}{|c|c|c|c|c|c|c|c|c|}
\hline & \multirow[b]{2}{*}{$\begin{array}{l}\text { Colic infants' } \\
\text { colic }\end{array}$} & \multirow[b]{2}{*}{$\begin{array}{l}\text { Colic infants' } \\
\text { fuss/cry }\end{array}$} & \multirow{2}{*}{$\begin{array}{l}\text { Persistent } \\
\text { criers' } \\
\text { fuss/cry }\end{array}$} & \multirow{2}{*}{$\begin{array}{l}\text { Moderatel } \\
\text { evening } \\
\text { criers' } \\
\text { fuss/cry }\end{array}$} & \multirow[b]{2}{*}{ Periods compared } & \multicolumn{2}{|c|}{ Manova } & \multirow[b]{2}{*}{$\begin{array}{l}\text { Duncan } M R \\
\text { test }\end{array}$} \\
\hline & & & & & & $F$ & $P$ & \\
\hline \multirow{3}{*}{$\begin{array}{l}\text { Diary (mean } \\
\text { period leng } \\
\text { in min) }\end{array}$} & & & & & Colic infants' colic: colic infants' fuss/cry & 5.5 & 0.036 & \\
\hline & $47(43)$ & $20(11)$ & $21(17)$ & $17(12)$ & $\begin{array}{l}\text { Colic infants' colic: persistent fuss/cry: } \\
\text { moderate/evening fuss/cry }\end{array}$ & 12.9 & 0.0000 & C $>$ PM \\
\hline & & & & & $\begin{array}{l}\text { Colic infants' fuss/cry: persistent fuss/cry: } \\
\text { moderate/evening fuss cry }\end{array}$ & 1.1 & 0.30 & NS \\
\hline \multicolumn{9}{|c|}{ Audio (mean (SD) } \\
\hline \multirow{2}{*}{$\begin{array}{l}\text { period len } \\
\text { in min) }\end{array}$} & $21(16)$ & $10(3)$ & $10(3)$ & $9(3)$ & $\begin{array}{l}\text { Colic infants' colic: persistent fuss/cry: } \\
\text { moderate/evening fuss/cry }\end{array}$ & 20.3 & 0.0000 & $\mathrm{C}>\mathrm{PM}$ \\
\hline & & & & & $\begin{array}{l}\text { Colic infants' fuss/cry: persistent fuss/cry: } \\
\text { moderate/evening fuss cry }\end{array}$ & 2.8 & 0.065 & $\mathbf{P}>\mathbf{M}$ \\
\hline
\end{tabular}

Colic infants: $n=14$; other persistent criers: $n=33$; other moderate/evening criers: $n=63$.

Diary: colic infants' colic periods: $n=26$; colic infants' non-colic fuss + /or cry periods: $n=118$; other persistent criers' fuss $+/$ or cry periods: $n=332$; other moderate/ evening criers' fuss + /or cry periods: $n=605$.

Audio: colic infants' colic periods: $n=40$; colic infants' non-colic fuss + /or cry periods: $n=204$; other persistent criers' fuss + /or cry periods: $n=653$; other moderate/ evening criers' fuss + /or cry periods: $n=1001$.

Table 5 Audible distress type of colic compared with non-colic periods

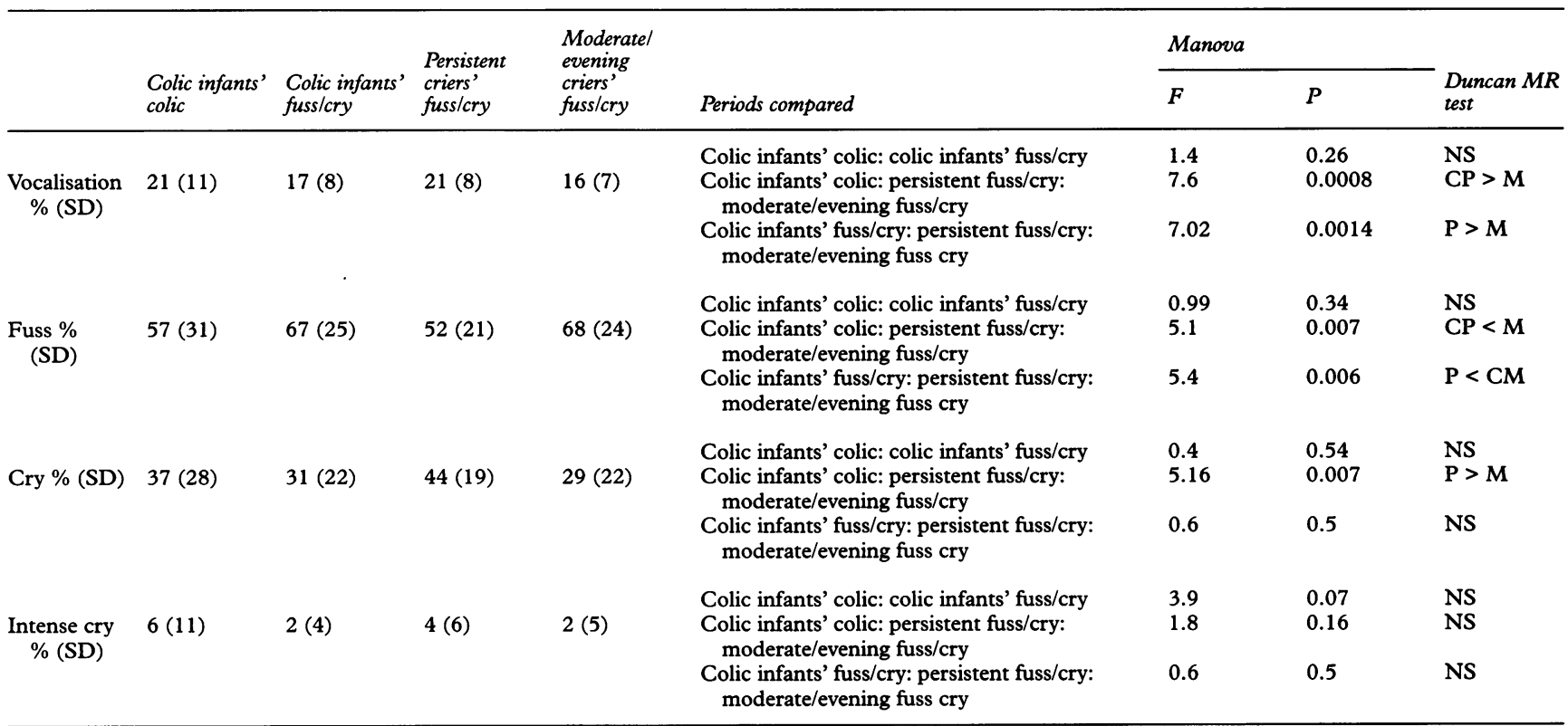

Colic infants: $n=14$; other persistent criers: $n=33$; other moderate/evening criers: $n=63$.

Colic infants' colic periods: $n=40$; colic infants' non-colic fuss $+/$ or cry periods: $n=204$; other persistent criers' fuss $+/$ or cry periods: $n=653$; other moderate/evening criers' fuss + /or cry periods: $n=1001$.

4). For each colic period identified, the matching audiorecording was searched to locate the audible distress periods that corresponded in time to the diary periods. Understandably, the onset times recorded in the diaries did not match the audiorecorded times exactly. However, the corresponding audiorecorded periods were identified without difficulty. Each distress period so identified was checked to ensure that an uninterrupted recording of audible distressed vocalisation had been successfully made.

As with the overall findings presented in table 1 , this search revealed more, and shorter, audiorecorded distress periods than reported in the diaries. A total of 40 audiorecorded distress periods corresponded in time to the 26 colic periods recorded on the diaries (table 4) To enhance the reliability of the data, each infant's colic and non-colic periods were aggregated to produce a single figure for each period type per infant. To simplify the findings, the audiorecording vocalisation measures were omitted, as they showed the same effects as the audiorecording period measures. Both diary and audiorecording methods identified the same infant effect: colic periods were significantly longer, both than the colicky babies' non-colic distress periods and the fuss/ crying periods of other babies (table 4). In contrast, there was no difference in length between the non-colic, fuss/crying, distress periods of colicky and other babies.

Table 5 examines the type of distress contained in the audiorecorded distress periods that corresponded to the diary colic, versus non-colic, periods. The colicky infants' noncolic periods again resembled those in moderate/evening criers. The proportion of audiorecorded intense crying is slightly greater in the periods identified as colic and, although the difference was not significant, it may be that a larger sample size would show a difference. However, the average proportions 
Table 6 Audible distress type at onset of colic compared with non-colic periods

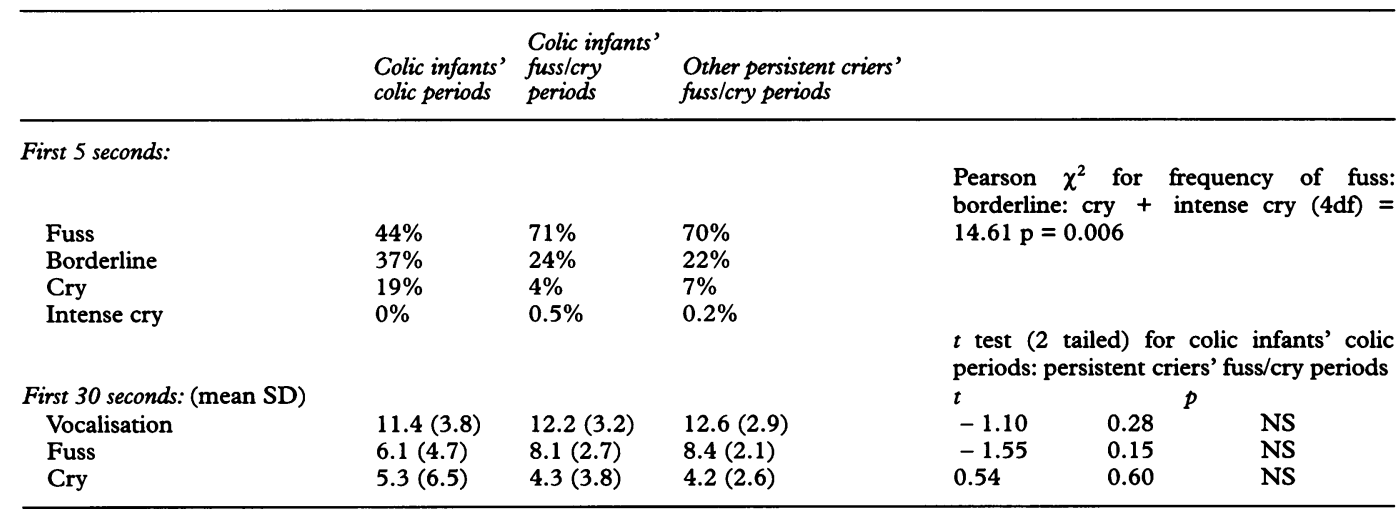

Colic infants: $n=11$; other persistent criers: $n=33$.

Colic infants' colic periods: $n=32$; colic infants' non-colic fuss $+/$ or cry periods $n=204$; other persistent criers' fuss $+/$ or cry periods: $\mathrm{n}=653$.

of intense crying were low- $6 \%$ or less-in all period types and infants. Both colicky infants' colic periods and non-colicky persistent criers' distress periods contained proportionately less fussing and more distressed vocalisation than other infants' distress periods. Contrary to what might be expected, however, only the non-colicky persistent criers'distress periods contained a significantly higher proportion of crying than the moderate/evening criers' distress periods.

The most striking finding was that the colic periods did not differ from the non-colic, fuss/
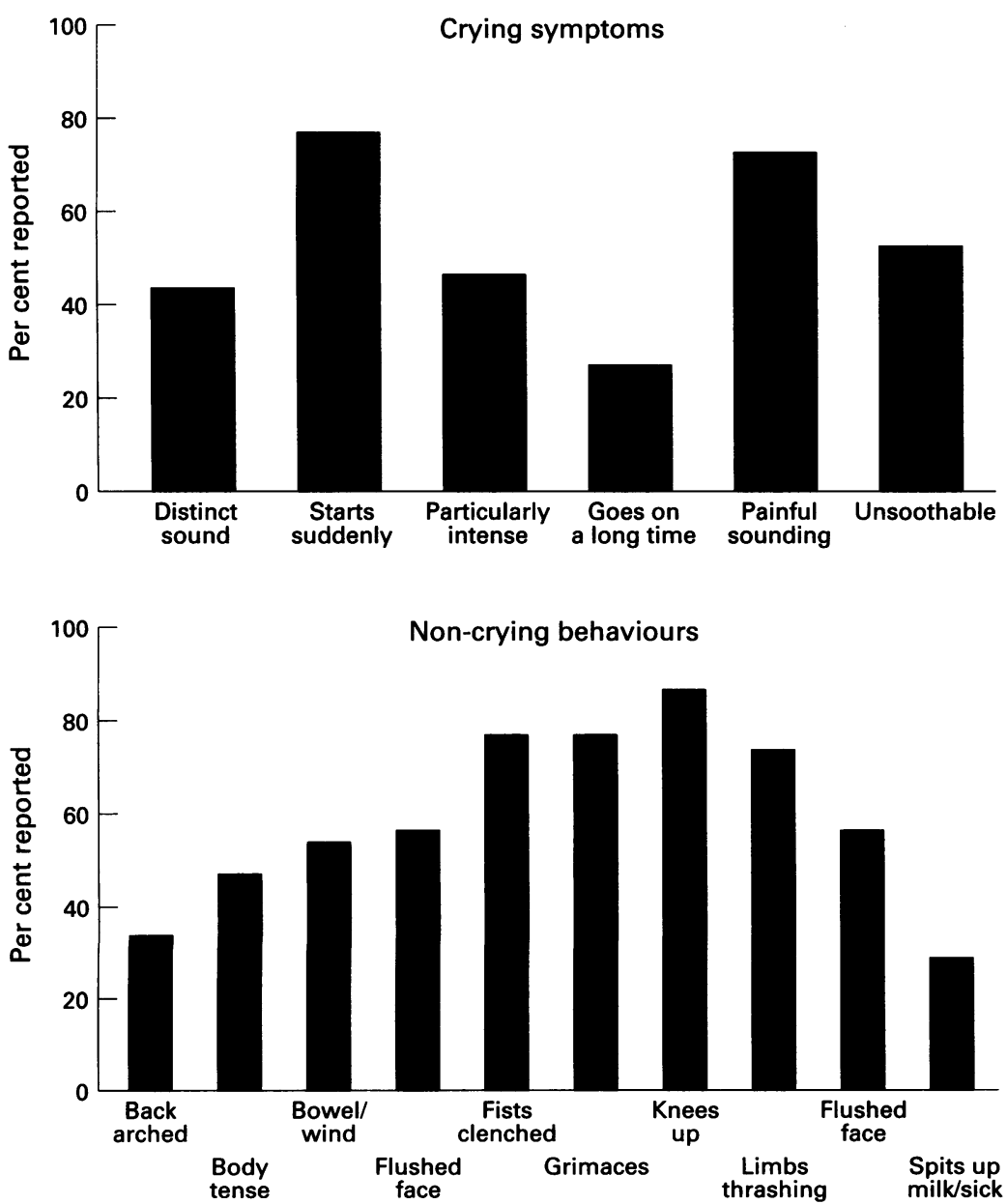

Figure 1 Behavioural symptoms checked most frequently by mothers, to indicate a bout of colic. crying, periods of persistent criers not reported to have colic on these indices of audible distress type. To ensure that the method used to recode borderline distress had not affected this finding, the data for fussing, borderline, and crying were also examined separately. No differences between the colic identified periods and the non-colic periods of persistent criers were found.

PAROXYSMAL ONSET?

As well as audible distress type, the word 'paroxysmal' that Wessel $e t$ al ${ }^{10}$ used to distinguish colic bouts, may denote other aspects of infants' distressed behaviour. Unfortunately, the ambiguity of this word makes such differences difficult to find. One pertinent question is whether colic bouts are distinguished by a sudden and intense onset, rather than a more gradual buildup in the intensity of distress. Even this question presents some pitfalls, because it is not certain that a subjectively perceived onset would necessarily correspond to the first few moments of vocal distress. Furthermore, as the audiorecordings made here were activated by infant vocalisation, previous non-vocal distress was missed. A further proviso is that, although a vocalisation starts the tape recorder almost instantaneously, the first few milliseconds were not recorded. None the less, to explore this question, table 6 compares the first 5 seconds, and first 30 seconds, of the colic periods with non-colic distress periods on the audiorecording measures of their distress type. There was little evidence of intense crying and no evidence that colic periods were especially likely to start with it. However, such periods were significantly less likely to include fuss, and more likely to include borderline or cry, in the first 5 seconds, than the non-colic distress periods of colicky infants or persistent criers. This difference ceased to be significant when the first 30 seconds of the periods were compared. The periods identified as colic did not contain a higher ratio of distressed vocalisation to pauses during the first 30 seconds. This finding was checked by examining the data for fuss, borderline, and crying 
separately. These analyses showed a nonsignificant tendency for the first 30 seconds of colic periods to have more crying.

In sum, these data indicate that colic periods tend to start with relatively intense distress vocalisation, but provide little support for the idea that trained listeners can distinguish the onset as demonstrably 'paroxysmal' in nature.

COLIC CHECKLIST DATA

Figure 1 shows the behavioural symptoms the mothers checked most frequently as the basis for their judgments that a bout of distress was colic. The figures given are for the 31 infants with diaries identifying colic bouts, but the findings for the 14 colicky infants with matching diaries and audiorecordings were essentially the same. For example, 11 of the 14 mothers identified 'sudden onset' and nine others 'painful sounding' as characteristics of the colic bouts recorded on the diary. Likewise, 12 of the 14 identified 'facial grimaces,' 13 'knees drawn up', and 12 'arms and legs thrashing' as features of colic. On average, the 14 mothers checked three (SD1) of six possible crying and eight (SD 2.9) of 17 other possible symptoms, with the 31 mothers showing very similar findings. These data indicate that the mothers based their judgments on a combination of audible and visible behaviours, with those listed above as the most common. Because of the ratings' homogeneity and the limited sample sizes, the checklist data cannot be mapped on to the matching audiorecordings with any degree of confidence. However, the colic periods of the 11 infants reported to have a sudden onset, were not discernibly different in their audible features from the bouts of the three infants not reported to have a sudden onset.

\section{Discussion}

Wessel et al's $^{10}$ widely used rule of threes definition of colic was based on the clinical observation that infants occasionally showed periods described as paroxysmal fussing. However, both the empirical study they used to substantiate their definition of colic as a clinical syndrome and the subsequent prolific published findings have been mainly based on maternal reports. Because distinguishing colic from normal fuss and crying requires a subjective judgment, it is remarkable that so little research has attempted to understand or substantiate the basis for such reports. Two somewhat distinct questions arise: (1) what distinguishes colic periods from other periods of fussing and crying?; (2) what is the basis for parental concerns and complaints to physicians about infant crying and colic?

Both the maternal diary reports and audiorecordings confirmed that the persistent criers differed from other infants in type, as well as amount, of distress. Their distress periods contained proportionately less fussing, which was matched by a proportionate increase in colic behaviour in the maternal diaries, and by a proportionate increase in crying, and in the ratio of distress vocalisation to pauses, in the audiorecordings.
These findings substantiate previous maternal diary and questionnaire reports that infants who cry prolonged amounts also cry relatively intensely. ${ }^{23}$ Zeskind $e t a l^{4}$ found that decreased pauses between distress vocalisations were associated with perceptions that the crying was more arousing and aversive for listeners. Although the group differences in vocalisation to pause ratios found here were not large, mothers of persistent criers may have perceived these characteristics. Equally important, however, is that most such mothers did not perceive their infants to show colic behaviour, while most infant distress in all three infant groups was considered by both mothers and researchers to be normal fuss and crying. Fussing was the principal behaviour for all three groups of infants, while crying made up about $25-45 \%$ of the infants' distress behaviour. Intense crying, defined as 'vocalisation which is both loud and frenzied sounding, conveying an urgent or imperative signal,' made up less than $5 \%$ of the audiorecorded infant distress and did not differ between the groups. Colic behaviour, defined as 'bouts of intense, unsoothable crying and other behaviour, perhaps due to stomach or bowel pain,' was rarely reported by the mothers, so that two thirds of the persistent criers were not reported as showing any colic behaviour.

These findings provide evidence that most infants selected using the Wessel rule of threes criteria exhibit distress that is normal in type. Such infants were distinguished by their amount of distress and increased ratio of crying to fussing, rather than by distress which was categorically different from normal fussing and crying.

Although this conclusion applies to most of the persistent criers studied, $35 \%$ of the persistent criers, $24 \%$ of the evening criers, and $7 \%$ of the moderate criers were reported by their mothers to have colic bouts. On average, one in six of these infants' distress periods was judged to be a colic bout. Analyses of the concurrent audiorecordings confirmed that the colic periods were particularly long in duration, a finding which also validates maternal diary findings elsewhere. ${ }^{73}$ In relation to distress type, however, the audiorecordings provided little evidence that the colic periods were different in their audible characteristics from the distress periods of other persistent criers not considered to have colic. The colic periods did not contain a higher ratio of distressed vocalisation to pauses. The proportions of fussing, crying, and intense crying resembled the proportions in the persistent criers'distress periods more generally, and just $6 \%$ of the audible distress in such periods was intense crying.

Although colic periods were more likely than non-colic periods to start with crying, they were not characterised by a strikingly paroxysmal onset so far as trained listeners could detect.

These findings can be explained in several ways. First the periods some mothers identified as colic bouts might have been considered normal, if long, periods of fussing and crying by 
other mothers. Secondly, the mothers' judgments may have been based on features of distress not detected by the audiorecording method used. The audiorecording findings were based on trained listeners' ability to distinguish specific atypical features and it is difficult, for example, to be sure whether the audible onset of a distress period corresponds to the maternally perceived onset. Although the colic bouts sounded like normal fussing and crying to the listeners, acoustic analyses, which examine fluctuations in vocal pitch or rhythm, might have been more revealing. ${ }^{25} \mathrm{~A}$ further possibility is that the mothers' perceptions were based on visible more than audible features of their infants' distress. The colic checklists completed by the mothers provide some support for this interpretation, as they indicated that the mothers used a combination of behaviours, including facial grimaces and limb movements, to distinguish colic bouts. Recent studies of infant pain indicate that it may be right to acknowledge such features. ${ }^{26}{ }^{27}$ Hadkistavropoulos $e t a l,{ }^{27}$ in particular, found that facial expression accounted for more of the variance in adults' judgments of newborn pain during injections than audible cries.

These findings emphasise the complex nature of the judgments involved in distinguishing the types and underlying causes of infant distress behaviour. ${ }^{28} \mathrm{~A}$ task for future research is to use videorecording and similar methods which will allow the reliability and validity of the mothers' judgments to be tested further. The present findings both provide some guidance and indicate the challenges which face such research. Although colic periods showed the same evening clustering as other distress, together with a somewhat specific tendency to follow feeds, many colic periods did not exhibit these regularities. Colic periods seem to be both rare and difficult to predict.

If colic behaviour is distinct, it clearly shares at least some features of normal infant distress, including evening clustering and a peak at around 6 weeks of age. The present failure to find evidence that colic bouts are audibly distinct, reinforces this similarity. Recently, Barr et al reported increased facial activity rates before (but not after) feeds in rule of threes infants, ${ }^{7}$ which they interpreted as evidence for colic as a distinct syndrome. However, although the rule of threes infants in Barr et al's study did show more facial activity (73 occurrences per minute) than controls (68 occurrences per minute), the difference was not large and, as the authors acknowledge, can also be interpreted as reflecting different degrees of distress. Theorists such as Lester and Zeskind ${ }^{24}$ have argued that infant distress varies along a single, graded continuum of increasing intensity or arousal. If this is correct, it could well be that the kinds of behaviour sometimes identified as colic are merely evidence of a high degree of distressed arousal, rather than of a discrete clinical condition. This interpretation is compatible with the idea that so-called colic behaviour is due to developmental adaptations which affect infants generally at this age, rather than to a gastrointestinal pathology. Although consistent with this formulation, the present findings do not provide any insight into the nature of the developmental processes involved. Studies which provide such insights, or which show whether colic type behaviour is elicited in other arousing circumstances, should help to delineate the conditions which produce this type of distress.

In view of the finding that colic bouts are rare, while most infant distress at this early age is fussing intermingled with crying, an intriguing question is whether colic behaviour itself is the principal reason why parents contact health professionals for infant crying related problems in the first three months. The findings here and elsewhere ${ }^{19}$ suggest that the audible character of the infants' distress may be less important than the fact that it is prolonged, hard to soothe, unpredictable and unexplained, so that mothers feel helpless and guilty in the face of such alarming and uncontrollable behaviour in young and vulnerable babies. Other studies have reported that mothers of firstborns are particularly likely to seek help for infant fussing and crying, although firstborns do not fuss and cry substantially more than babies born subsequently, suggesting that parental inexperience may also be a contributing factor. ${ }^{29}$ In a study of infants referred to physicians for problem crying, Barr et $a l^{7}$ found that the parents considered their babies' distress to be 'sick sounding,' even though two thirds of the infants did not fuss and cry more than their control group norm. It seems likely that individual parents' worries, together with cultural expectations about normal baby behaviour, contribute to the clinical picture. These findings emphasise the need to distinguish between infant behaviour and parent perception and concern about crying as the target for therapeutic work. ${ }^{18}$

Lastly, the findings throw some light on to the issue of the prevalence of infant colic. The estimated prevalence of colic has varied widely from $10 \%$ to $40 \% .^{83031}$ So far as colic is defined by the amount of infant fussing and crying, it is likely that a relatively high prevalence will result. For example, $23 \%$ of the community sample screened for this study met the Wessel criteria for fuss/crying amount, although only a third of these infants were reported by their mothers to show colic behaviour as a distinct type of distress. As well as the amount and type of infant distress, colic has frequently been defined by parental complaint about problem crying. ${ }^{72}$ While none of these definitions is inherently superior, the prevalence of colic must depend on the definition used, so that it is important that this is specified in future research.

This study was supported by Project Grant 037089 from The Wellcome Trust. Eric Hadley designed and constructed the audiorecording equipment. Sandy Barker helped with recruitment, Amanda Eve with audiotranscription, and Charlie Owen with computing. We are grateful to David Harvey and the staff at Queen Charlotte's and Chelsea Hospital and to the families who took part.

1 Forsyth BW, Leventhal JM, McCarthy PL Mothers' perceptions of problems of feeding and crying behaviour. $A m \mathcal{F}$ Dis Child 1985; 139:269-72. 
2 St James-Roberts I, Halil T. Infant crying patterns in the first year: normal community and clinical findings. $\mathcal{f}$ Child Psychol Psychiatry 1993;32:951-68.

3 Wilkie CF, Ames EW. The relationship of infant crying to parental stress in the transition to parenthood. $\mathcal{F}$ Marriage Fam 1986;48:545-50.

4 Frodi AM. Contribution of infant characteristics to child abuse. Am $\mathcal{F}$ Ment Defect 1981; 85:341-9.

5 St James-Roberts I, Hurry J, Bowyer J. Objective confirmation of crying durations in infants referred for excessive crying. Arch Dis Child 1993;68:82-4.

6 Treem WR, Hyams, JS, Blankschen E, et al. Evaluation of the effect of a fiber-enriched formula on infant colic. $\mathcal{F}$ Pediatr 1991;119:695-701.

7 Barr RG, Rotman A, Yaremko J, et al. The crying of infants with colic: a controlled empirical description. Pediatrics 1992;90:14-21.

8 Carey WB. 'Colic' - primary excessive crying as an infant-environment interaction. Pediatr Clin North $\mathrm{Am}$ infant-environment

9 Illingworth RS. Infantile colic revisited. Arch Dis Child 1985;60:981-5.

10 Wessel MA, Cobb JC, Jackson EB, et al. Paroxysmal fussing in infancy, sometimes called 'colic'. Pediatrics 1954;14:42133.

11 Barr RG. Colic and gas. In: Zelazo PR, Barr RG, eds. Pediatric gastrointestinal disease: Pathophysiology, diagnosis and management. Hillsdale: Lawrence Erlbaum Associates, 1989:93-121.

12 Brazelton TB. Neonatal behavioral assessment scale. 2nd Edn. London: Blackwell Scientific Medical Publications, 1984.

13 Hunziker UA, Barr RG. Increased carrying reduces infant crying: a randomised control trial. Pediatrics 1986;77:641-

14 Emde RN, Gaensbauer TJ, Harmon TJ. Emotional expression in infancy: a biobehavioral study. New York: International in infancy: a biobehavioral

15 Alvarez M, St James-Roberts I. Infant fussing and crying patterns in an urban community in Denmark. Acta Paediatr 1996;85: 463-6.

16 Barr RG, Konner M, Bakeman R, Adamson L. Crying in Kung San infants: a test of the cultural specificity hypothesis. Dev Med Child Neurol 1991;33:601-10.

17 St James-Roberts I, Bowyer J, Varghese S, Sawdon J. Infant crying patterns in Manali and London. Child Health Care Dev 1994;20:323-37.
18 St James-Roberts I. Managing infants who cry persistently. BMF 1992;304:997-8.

19 St James-Roberts I, Conroy S, Wilsher K. Clinical, developmental and social aspects of infant crying and colic. Early mental and social aspects of
Dev Parent 1995;4:177-89.

20 Lester BM, Boukydis CFZ, Garcia-Coll C, et al. Infantile colic: acoustic cry characteristics, maternal perception of cry, and temperament. Infant Behav Dev 1992;15:15-26.

21 Barr RG, Desilets J. The normal crying curve: hoops and hurdles. In: Lester B, Newman J, Pederson F, eds Biological and social aspects of infant crying. New York: Plenum, 1996 (in press).

22 Barr RG, Kramer MS, Boisjoly C, et al. Parental diary of infant cry and fuss behaviour. Arch Dis Child 1988;63:380-

23 Stifter CA, Braungart J. Infant colic: a transient condition with no apparent effects. F App Dev Psychol 1992;13:44762.

24 Zeskind PS, Klein L, Marshall TR. Adults' perceptions of experimental modifications of durations of pauses and expiratory sounds in infant crying. Dev Psychol 1992;28: 1153-162.

25 Lester BM, Zeskind PS. A biobehavioral perspective on crying in early infancy. In: Fitzgerald H, Lester BM, Yogman New York: Plenum Press; 1982:133-80.

26 Craig KD, Hadkistavropoulos HD, Grunau RVE, Whitfield MF. A comparison of two measures of facial activity during pain in the newborn child. $\mathcal{f}$ Pediatr Psychol 1994;19:305pain

27 Hadkistravlopoulos HD, Craig KD, Grunau RVE, Johnston CC. Judging pain in newborns: facial and cry determinants. F Pediatr Psychol 1994;19:485-91.

28 Barr RG. Is this infant pain?: caveats from the clinical setting. Am Pain Soc F 1992;1:187-90.

29 Michelsson K, Rinne A, Paaganen S. Crying, feeding and sleeping patterns in 1 to 12 month-old infants. Child Care Health Dev 1990;16:99-111.

30 Rubin SP, Prendergast M. Infantile colic: incidence and treatment in a Norfolk community. Child Health Care Dev 1984;10:219-26.

31 Stahlberg MR. Infantile colic: occurrence and risk factors. Eur $\mathcal{F}$ Pediatr 1984;143:108-11.

32 Rautava P, Helenius H, Lehtonen L. Psychosocial predisposing factors for infantile colic. BMf 1993;307:600-4. 